\title{
Place de la biologie moléculaire des hémopathies malignes pour le réanimateur
}

\section{Molecular Biology of the Hematology Neoplasms: What should know the Intensivist?}

\author{
É. Lengliné \\ Reçu le 8 mars 2016; accepté le 9 mars 2016 \\ (C) SRLF et Lavoisier SAS 2016
}

Les progrès techniques du diagnostic biologique des dernières décennies ont permis d'une part de faire progresser la compréhension de l'ontologie des hémopathies malignes mais surtout un formidable essor de la recherche « translationnelle » responsable d'une amélioration constante des stratégies thérapeutiques.

Les différentes hémopathies résultent de la transformation cancéreuse d'une cellule normale (notion de clonalité : toutes les cellules cancéreuses ont la même origine) faisant suite à la survenue d'altérations génétiques touchant des mécanismes importants de la vie cellulaire. Ces altérations peuvent êtres de toute taille (du chromosome entier à quelques nucléotides) et de tout type (gains, pertes de matériel génétique, anomalies de structure de l'ADN). Les plus grosses de ces anomalies (plusieurs méga-bases) n'ont pu être mises en évidence par l'examen du caryotype en métaphase des cellules malignes qu'à partir du milieu des années 1970. En effet, historiquement, le diagnostic des différentes hémopathies était seulement fondé sur la morphologie (aspect au microscope des tissus ou des cellules) à l'origine des premières classifications, dissociées de toute notion de physiopathologie et d'intérêt de stratification thérapeutique [1].

Les nouvelles techniques de biologie moléculaire ont permis de transformer ce paradigme d'un travail de quasibotaniste en un outil de prise en charge indispensable au quotidien. L'objet de cet éditorial est d'en appréhender les éléments basiques nécessaires au réanimateur en répondant à quatre questions simples.

É. Lengliné $(\bowtie)$

Service d'hématologie adulte,

hôpital Saint-Louis,

AP-HP, 1 avenue Claude Vellefaux,

F-75010 Paris

e-mail : etienne.lengline@aphp.fr

\section{Quelles sont les moyens d'analyses moléculaires d'une hémopathie maligne?}

Les examens de biologie moléculaire recouvrent différentes techniques actuellement utilisées en routine médicale. L'outil de base est la PCR permettant d'amplifier un fragment d'ADN (de quelques centaines de bases) entre deux amorces dont la séquence est connue. La portion d'intérêt située entre les deux amorces peut ensuite être séquencée par technique Sanger afin de détecter des mutations somatiques spécifiques de la tumeur. La fabrication d'amorces et de sondes fluorescentes spécifiques de séquences permet de quantifier précisément le nombre de copies d'ADN présentes initialement avant la réaction de PCR (PCR quantitative) et, dans le cas d'une mutation oncogénique, est généralement bien corrélée à la quantité de cellules tumorales.

Des pertes ou gains de plus grandes tailles peuvent être identifiés par des techniques d'hybridation génomique comparative ( $C G H$ array » : on hybride comparativement sur une puce de l'ADN tumoral et de l'ADN de référence afin d'identifier des déséquilibres), cette technique permettant d'avoir une résolution beaucoup plus importante que le caryotype standard mais ne détectant pas les anomalies de structure équilibrées.

Enfin, des nouvelles techniques de séquençages directs (NGS) sont de plus en plus utilisées et ont comme principal avantage la possibilité de séquencer l'ADN sans passer par une étape préalable de PCR, ce qui permet un gain considérable de débit. Aussi le séquençage simultané par NGS de plusieurs dizaines de gènes d'intérêt pronostique ou thérapeutique devient de plus en plus courant dans un certain nombre d'hémopathies. Ce type de technique permet aussi d'avoir une idée de la structure sous-clonale des tumeurs car elle permet une quantification des taux de mutations en fonction du nombre de molécules d'ADN de la portion d'intérêt séquencée (profondeur corrélée au nombre de molécules séquencées : « reads ») 


\section{Dans quel but faire des analyses de biologie moléculaire?}

Actuellement les analyses de biologie moléculaires sont utilisées en pratique courante pour cinq principales indications décrites ici dans leur ordre d'importance clinique.

\section{Prédire la sensibilité de la maladie aux traitements.}

La détection de mutations somatiques récurrentes de certains gènes est actuellement utilisée pour stratifier les stratégies thérapeutiques. En effet, si la plupart des mutations n'ont pas d'influence sur l'évolution de la maladie traitée, dans certains cas, des analyses ciblées de certains gènes permettent de mieux définir le pronostic de certaines hémopathies et de modifier le schéma thérapeutique pour améliorer le devenir des patients. Dans les leucémies aiguës myéloïdes (LAM), la probabilité de guérison par chimiothérapie intensive est essentiellement basée sur l'âge et le caryotype. Néanmoins, environ la moitié des LAM ont un caryotype normal et un pronostic intermédiaire. Il a été montré de façon constante et indépendante que l'analyse des mutations des trois gènes NPM1, FLT3 et CEBPA permettait de prédire le risque de rechute et de guérison des patients traités par chimiothérapie [2-5]. Dans le cas précis de ces mutations, le risque peut être diminué par la réalisation d'une allogreffe de moelle chez les sujets les plus jeunes. Il est important de bien comprendre que les facteurs pronostiques moléculaires sont très dépendants du traitement appliqué et ne doivent être vus que comme des facteurs de réponse à celui-ci. Dans les leucémies aiguës lymphoblastiques (LAL) de la lignée $\mathrm{T}$ de l'adulte, les mutations de quatre gènes (NOTCH1, FBXW7, RAS, PTEN) peuvent prédire de façon importante le risque de rechute de patients traités par un schéma intensif de type pédiatrique (Group for Research in Adult Acute Lymphoblastic Leukemia, GRAALL) et identifient un groupe de patient (plus de la moitié des cas) avec une survie estimée à plus de $90 \%$. Pourtant les patients ayant un génotype défavorable dans ce contexte n'ont pas moins de risque de rechute après allogreffe et ces analyses ne servent donc pas à l'heure actuelle à définir son indication. La Fig. 1 résume les gènes d'intérêt actuellement utilisés couramment.

\section{Analyser la sensibilité in vivo au traitement}

Comme mentionné plus haut, il est possible de tirer parti de l'origine clonale des tumeurs cancéreuses pour identifier des caractéristiques moléculaires spécifiques du clone tumoral afin de le détecter au cours des différentes phases de traitement. La vitesse de diminution de la maladie et notamment la quantité restante de maladie après l'obtention d'une rémission complète (maladie résiduelle minimale « $\mathrm{MRD} »)$ dans les LAL est devenu le facteur prédictif principal du risque de rechute et de mortalité surpassant toutes les autres caractéristiques initiales [6,7]. Une PCR quantitative spécifique de la région variable du gène des immunoglobulines ou du TCR est utilisée pour le suivi de ces hémopathies. Une maladie résiduelle supérieure à $10^{-4}$ un à deux mois après le début des chimiothérapies de consolidation est généralement reconnue comme de mauvais pronostic. Une allogreffe de moelle et/ou de nouvelles thérapies immunologiques permettraient vraisemblablement de diminuer ce risque. Dans les LAM, l'analyse de la maladie résiduelle est moins aisée. Il est possible de quantifier les transcrits de fusion ou les mutations, notamment de NPM1, dont la décroissance semble également avoir un impact pronostique, néanmoins moins fort que dans les LAL. Enfin, dans les leucémies myéloïdes chroniques (LMC), la survenue d'une transformation et la survie à long terme sont également liées à la diminution du taux de transcrit de fusion BCR-ABL sous inhibiteur de tyrosine kinase [8].

\section{Faire un diagnostic correct.}

Le diagnostic des hémopathies malignes reste dans l'immense majorité des cas basée sur la morphologie des cellules analysées sur un frottis de sang, de moelle ou de tissu. Parfois, des analyses de biologie moléculaire permettent de redresser un

\begin{tabular}{llll}
\hline LAM & LAL-B & LAL-T & SMD \\
\hline \hline NPM1 (si FLT3 GL) & IKZF1 & NOTCH1 & TP53 \\
FLT3-ITD & TP53 & FBXW7 & \\
CEBPA (2 allèles) & & RAS & \\
TP53 & & PTEN & \\
DNMT3A & & & \\
\hline \hline
\end{tabular}

Fig. 1 Exemples de mutations de gènes utiles à la stratification thérapeutique dans diverses hémopathies malignes (vert : favorable; rouge : néfaste). NPM1 : nucleophosmin ; FLT3 : fms like tyrosine kinase 3 ; CEBPA : CCAAT/enhancer-binding protein alpha ; DNMT3A : DNA methyl-transferase 3A ; IKZF1 : Ikaros family zinc finger protein 1 ; NOTCH1 : Notch homolog 1 ; FBXW7 : F-box and WD repeat domain containing 7 ; PTEN : Phosphatase and TENsin homolog ; GL : germline ; ITD internal tandem duplication ; LAL : leucémie aiguë lyphoblastique ; SMD : syndrome myélodysplasique 
diagnostic douteux, notamment dans certaines hémopathies lymphoïdes indolentes (mutations de BRAF dans les tricholeucémies) ou d'affirmer le diagnostic de syndrome myélodysplasiques (mutations du splicéosome, ou de gènes impliqués dans l'épigénétique).

\section{Identifier des voies oncogéniques pouvant être ciblées par des traitements}

À l'instar des inhibiteurs de tyrosine kinases dans la LMC, certaines mutations peuvent êtres les cibles de traitements dans d'autres hémopathies. Aussi des inhibiteurs de FLT3 dans les LAM sont actuellement en cours de développement et nécessitent d'en connaître le statut mutationnel. De la même façon, des mutations de kinases (ABL1, PDGFR, JAK, EPOR) peuvent êtres retrouvées dans des LAL-B (dites « Ph1 like ») et les cibles d'inhibiteurs. Des médicaments ciblant les mutations d'IDH1 et IDH2 (10 à $20 \%$ des cas) sont également très prometteurs dans les traitements de LAM et amenés à prendre de l'importance dans le futur, rendant nécessaire leur identification précoce.

\section{Identifier des mutations germinales prédisposant à des pathologies hématologiques}

Enfin, la mutation de certains gènes peut parfois être retrouvée au niveau germinal (dans toutes les cellules de l'individu et non pas uniquement dans le clone tumoral) et prédisposent à l'apparition de cancers hématologiques. Ces anomalies sont importantes car elles peuvent guider non seulement le conseil génétique éventuel mais aussi les décisions thérapeutiques individuelles et la sélection d'un donneur de moelle apparenté non muté. C'est le cas par exemple des familles avec mutation de RUNX1 ou de GATA2 ayant une propension importante à développer des hémopathies myéloïdes

\section{Quels sont les nécessités techniques ?}

Les analyses sus-mentionnées, pour être utilisées sans problème, nécessitent la présence au laboratoire de matériel tumoral de bonne qualité et le plus souvent avant tout traitement. Ceci est d'une importance particulière en réanimation où une prise en charge spécifique de l'hémopathie peut être nécessaire en urgence. Si l'accessibilité de la tumeur ne pose généralement pas de problème pour une leucémie aiguë, a fortiori si une grande quantité de cellules tumorales est circulante (LA hyperleucocytaire), elle peut être plus difficile pour un d'autres hémopathies malignes (lymphome lymphoblastique T médiatisnal, LAM pancytopénique, sarcome granulocytaire) pour lesquels une ponction/biopsie guidée de bonne taille est nécessaire. Par ailleurs, une bonne coordination avec le laboratoire d'hématologie est cruciale car si l'ADN est une molécule assez stable ne soufrant pas d'une « attente d'une nuit sur la paillasse », l'ARN peut se dégrader rapidement en l'absence de congélation immédiate et un défaut de conservation peut compromettre certaines analyses.

Une fois le prélèvement arrivé au laboratoire, le délai de rendu est généralement long, de plusieurs jours voire semaines, en raison du temps requis pour les différentes étapes techniques. Ainsi, lors de la prise en charge d'une hémopathie inaugurale en réanimation, les résultats de biologie moléculaire ne pourront pas être disponibles et influer sur la prise en charge hématologique.

\section{Comment ces différents paramètres peuvent interférer avec la prise en charge en réanimation}

Les points mentionnés plus haut expliquent les utilisations faites à l'heure actuelle des analyses de biologie moléculaire par les hématologues. En réanimation, le plus souvent, la situation clinique ne justifie pas l'utilisation de ces indicateurs dans l'algorithme décisionnel. Encore une fois, plusieurs situations peuvent être identifiées.

\section{Réanimation d'une complication inaugurale d'une hémopathie}

- Ne pas négliger les prélèvements tumoraux : entre un tiers et la moitié des patients admis en réanimation avec une hémopathie maligne le sont au moment du diagnostic [9]. Dans cette situation, il appartient au réanimateur d'effectuer le nécessaire pour effectuer le diagnostic. La qualité du prélèvement tumoral est primordiale pour les décisions ultérieures qui résulteront des analyses de biologie moléculaire. Un prélèvement ne permettant qu'une analyse morphologique, même s'il apporte le diagnostic, risque de porter préjudice au patient pour les décisions thérapeutiques ultérieures. Il est donc indispensable de sécuriser avant tout traitement le circuit des prélèvements, le plus simple étant d'obtenir un contact téléphonique avec le biologiste avant le prélèvement diagnostique (type de tube, moyen et température d'envoi, type de conservation à prévoir en cas d'horaire non ouvrable, etc.) Aussi, un deuxième contact est recommandé après le prélèvement afin de s'assurer de la bonne réception, de la quantité et qualité de cellules tumorales suffisantes pour les différents secteurs du laboratoire.

- ne (quasi) rien en attendre pour la prise en charge en réanimation : concernant la prise en charge thérapeutique, les analyses de biologie moléculaire, en plus de ne pas être disponibles immédiatement, n'ont pas d'impact sur 
l'apparition ni le pronostic des détresses vitales. Au cours des LAM, les mutations de NPM1 ou FLT3 sont plus souvent associées a des formes initialement hyperleucocytaires et donc à risque de syndrome de lyse tumorale, de leucostase, de CIVD, etc. Mais dans cette situation, le pronostic en réanimation reste guidé par l'apparition des complications en elles-mêmes et par le nombre de défaillance(s) vitale(s) $[10,11]$.

\section{Hémopathie en cours de traitement. Décider de l'intensité du projet de soins}

Dans la situation où un patient est admis en réanimation au cours du traitement de son hémopathie déjà complètement caractérisée, toutes les analyses disponibles peuvent être utilisées pour avoir une idée du caractère réaliste d'un projet hématologique curatif. Il faut néanmoins bien garder à l'esprit que les résultats d'analyses moléculaires sont loin d'être des facteurs pronostiques idéaux et s'ils permettent de guider des décisions thérapeutiques tels qu'un changement de chimiothérapie ou une greffe de moelle, ils ne peuvent pas définir le pronostic individuel avec certitude. Aussi les décisions d'admission ou la définition de l'intensité des soins en réanimation doit rester une équation multiparamétrique basée non seulement sur les caractéristiques de l'hémopathie mais aussi (et surtout) sur : l'âge du patient, son état général et nutritionnel, la réversibilité prévisible de la complication aiguë et enfin la quantité de défaillance(s) d'organe(s) et leur persistance en cours de réanimation [10].

Liens d'intérêts : L'auteur déclare ne pas avoir de lien d'intérêt.

\section{Références}

1. Bennett JM, Catovsky D, Daniel MT, et al (1976) Proposals for the classification of the acute leukaemias. French-American-British (FAB) co-operative group. Br J Haematol 33:451-8

2. Yanada M, Matsuo K, Suzuki T, et al (2005) Prognostic significance of FLT3 internal tandem duplication and tyrosine kinase domain mutations for acute myeloid leukemia: a meta-analysis. Leukemia 19:1345-9

3. Thiede C, Koch S, Creutzig E, et al (2006) Prevalence and prognostic impact of NPM1 mutations in 1485 adult patients with acute myeloid leukemia (AML). Blood 107:4011-20

4. Renneville A, Boissel N, Gachard N, et al (2009) The favorable impact of CEBPA mutations in patients with acute myeloid leukemia is only observed in the absence of associated cytogenetic abnormalities and FLT3 internal duplication. Blood 113:5090-3

5. Patel JP, Gonen M, Figueroa ME, et al (2012) Prognostic relevance of integrated genetic profiling in acute myeloid leukemia. The New England journal of medicine 366:1079-89

6. Bruggemann M, Raff T, Kneba M (2012) Has MRD monitoring superseded other prognostic factors in adult ALL? Blood 120:4470-81

7. Beldjord K, Chevret S, Asnafi V, et al (2014) Oncogenetics and minimal residual disease are independent outcome predictors in adult patients with acute lymphoblastic leukemia. Blood 123:3739-49

8. Baccarani M, Deininger MW, Rosti G, et al (2013) European LeukemiaNet recommendations for the management of chronic myeloid leukemia: 2013. Blood 122:872-84

9. Azoulay E, Mokart D, Pene F, et al (2013) Outcomes of critically ill patients with hematologic malignancies: prospective multicenter data from France and Belgium--a "groupe de recherche respiratoire en réanimation onco-hématologique" study. J Clin Oncol $31: 2810-8$

10. Azoulay E, Pene F, Darmon M, et al (2015) Managing critically ill hematology patients: Time to think differently. Blood Rev 29:359-67

11. Mamez AC, Raffoux E, Chevret S, et al (2016) Pre-treatment with oral hydroxyurea prior to intensive chemotherapy improves early survival of patients with high hyperleukocytosis in acute myeloid leukemia. Leuk Lymphoma 5:1-8 